EPJ Web of Conferences 107, 07003 (2016)

DOI: $10.1051 /$ epjconf/201610707003

(C) Owned by the authors, published by EDP Sciences, 2016

\title{
Energy dependence of fission observables
}

\author{
Horia Paşca ${ }^{1,2, a}$ \\ ${ }^{1}$ Joint Institute for Nuclear Research, 141980 Dubna, Russia \\ 2 "Babeş-Bolyai" University, 400084 Cluj-Napoca, Romania
}

\begin{abstract}
The mass, charge and isotopic distributions of fission fragments are studied within an improved scission-point statistical model in the reaction ${ }^{235} \mathrm{U}+n$ at different energies of the incident neutron. The available experimental data are well reproduced and the energy-dependencies of the observable characteristics of fission are predicted for future experiments. The calculated mass distribution of ${ }^{238} \mathrm{U}+n$ is also compared with experimental data.
\end{abstract}

\section{Introduction}

It is well-known that the mass (charge) distributions of the neutron induced fission of pre-actinides are symmetric, while of the nuclei U-Cf and Ac-Pa near the line of stability are asymmetric with two and three maxima, respectively $[1,2]$. For the neutron-deficient actinides Ac$\mathrm{U}$, the symmetric mode is the most predominant. However, in neutron-deficient ${ }^{180} \mathrm{Hg}$ the asymmetric mass distribution of fission fragments was unexpectedly observed in the recent experiment $[1,3]$. Despite six decades of the experimental and theoretical research there is still no satisfactory understanding why the transition from a symmetric to an asymmetric fission occurs with increasing mass or decreasing excitation energy of the fissioning nucleus. In Ref. [4], it was suggested that the competition between symmetric and asymmetric fission is related to the shell effects in the deformed fissioning nucleus. With increasing energy the shell effects wash out, leaving the nucleus with a dominant symmetric mode of fission. However, the new experimental data of fission of ${ }^{180} \mathrm{Hg}$ show that the asymmetric mass distribution cannot be anticipated from the microscopic effects in the fragments, which are weak here and do not survive near the saddle. The experimental nuclear-charge distributions of fission fragments of nuclei At-U with $A<220$ do not seem to show any clear signature of the shell effects [2]. The peak-to-valley ratio and the even-odd effect in the charge distributions in the electro-magnetic-induced fission of ${ }^{233,234} \mathrm{U}$ can not be quantitatively understood on the basis of previously measured thermal-neutron-induced data of the excitation-energy dependence of fission characteristics [2]. So, further theoretical and experimental investigations of the mass- and energy-dependence are required.

Another longstanding question is at which point of the fission path the mass (charge) and energy distributions of

\footnotetext{
${ }^{\mathrm{a} e-m a i l: \text { pasca@ } \mathrm{theor} . j i n r}$.ru
}

the fission fragments are determined. There exist several versions of "saddle-point models" and "scission-point models" which put this crucial point closer to the saddleor scission-point, where the stability properties of the fragments become active [4-9]. There is also a dynamical approach [10] where the potential energy surface in the entire deformation range from the saddle-point to scission-point governs the final yields. However, the main problem of this approach is the impossibility of calculation of the energy distribution. The detailed analysis indicates that the formation of the experimentally observed fragment total kinetic energy- and mass-distributions occurs in the region closer to the scission region than to the saddle-point. As shown in Ref. [10], the mass-asymmetry distribution reflects the structure of the potential-energy surface in the scission region. In this region, the neck becomes well developed and the fragments attain their individual character. With the saddle-point model the experimental data of fission of actinides and pre-actinides: mass, charge, energy, and neutron multiplicity distributions were well described $[6,9]$. The wide range of described fission observables and effects demonstrated the predictive power of such type the models.

Although the fission product mass distributions have been essentially compiled for the fission of ${ }^{235} \mathrm{U}$ by thermal neutrons, relatively little data are available on the characteristics of mass distributions for monoenergeticneutron-induced fission, particularly as a function of incident neutron energy $E_{n}$. In this paper, we will study the evolution of the shape of charge and mass distributions of the fission fragments for the reactions ${ }^{235} \mathrm{U}(n, \mathrm{f})$ and ${ }^{238} \mathrm{U}(n, \mathrm{f})$ with increasing incident energy of neutron from the thermal energy up to $55 \mathrm{MeV}$. As is well-known, the thermal-neutron-induced fission products of nuclei ${ }^{236} \mathrm{U}$ and ${ }^{239} \mathrm{U}$ have the two humped charge and mass distributions. The fission observables will be described in the statistical way employing the dinuclear system (DNS) model $[6,9,11]$ which is the improved scission-point approach. 
The main ingredient of our description is the sophisticated potential energy as a function of mass (charge) asymmetry, deformations of nuclei, and internuclear distance. The knowledge of the deformations of the nascent DNS fragments is crucial at the moment of scission.

This article is organized as follows: in Sec. 2 we will present our model; the results of calculations will be discussed in Sec. 3 and in Sec. 4 we will give a brief summary.

\section{Model}

The statistical scission-point model relies on the assumption that the statistical equilibrium is established at scission and the observable characteristics of fission process are formed near the prescission configurations of the fissioning nucleus. The DNS model is well suited for describing the scission configuration, which consists of two well defined fission fragments. The fissioning nucleus at scission point is modeled by two nearly touching coaxial spheroids - fragments of the DNS with masses (charges) numbers $A_{L}\left(Z_{L}\right)$ and $A_{H}\left(Z_{H}\right)$ for the light $(L)$ and heavy $(H)$ fragments, respectively. The $A=A_{L}+A_{H}(Z=$ $Z_{L}+Z_{H}$ ) is the mass (charge) number of fissioning nucleus. By taking into consideration the volume conservation, the shape of the system is defined by the mass and charge numbers of the fragments, deformation parameters of fragments, $\beta_{i}(i=L, H)$, and the inter-fragment distance $R$. The index $i$ designates the light or heavy fragment of the DNS. The potential energy

$$
\begin{aligned}
U\left(A_{i},\right. & \left.Z_{i}, \beta_{i}, R\right)= \\
& =U_{L}^{L D}\left(A_{L}, Z_{L}, \beta_{L}\right)+\delta U_{L}^{\text {shell }}\left(A_{L}, Z_{L}, \beta_{L}, E_{L}^{*}\right)+ \\
& +U_{H}^{L D}\left(A_{H}, Z_{H}, \beta_{H}\right)+\delta U_{H}^{\text {shell }}\left(A_{H}, Z_{H}, \beta_{H}, E_{H}^{*}\right)+ \\
& +V^{C}\left(A_{i}, Z_{i}, \beta_{i}, R\right)+V^{N}\left(A_{i}, Z_{i}, \beta_{i}, R\right)
\end{aligned}
$$

of the DNS consists of the energies of the fragments and energy of their interaction. The energy of each fragment consists of the liquid-drop (LD) energy $U_{i}^{L D}$ and deformation dependent shell-correction term $\delta U_{i}^{\text {shell }}$. The symmetry, Coulomb and surface liquid-drop parts for cold nuclei are calculated as in [6]

$$
\begin{aligned}
& U_{i}^{\text {sym }}\left(A_{i}, Z_{i}\right)=27.612 \frac{\left(N_{i}-Z_{i}\right)^{2}}{A_{i}}, \\
& U_{i}^{C}\left(A_{i}, Z_{i}, \beta_{i}\right)=\frac{3}{5} \frac{\left(Z_{i} e\right)^{2}}{R_{0, i}} \frac{\beta_{i}^{1 / 3}}{\sqrt{\beta_{i}^{2}-1}} \ln \left(\beta_{i}+\sqrt{\beta_{i}^{2}-1}\right) \\
& U_{i}^{\text {Surf }}\left(A_{i}, Z_{i}, \beta_{i}\right)=\sigma_{i} S_{i}
\end{aligned}
$$

with the surface area of the deformed spheroid $S_{i}$ and surface tension $\sigma_{i}=\sigma_{0, i}\left(1+k_{i}\left(\beta_{i}-\beta_{i}^{g . s .}\right)^{2}\right)$, $\left.\sigma_{0, i}=0.9517\left(1-1.7826\left(\left(N_{i}-Z_{i}\right)^{2}\right) / A_{i}\right)^{2}\right)$ and $k_{i}=$ $1 /\left[1+\exp \left[-0.063\left(C_{v i b}\left(Z_{i}, A_{i}\right)-67\right)\right]\right]$. Here $\beta_{i}^{g . s .}$ and $k_{i}$ are the ground state deformation and the surface stiffness parameter.

The temperature dependence of the LD terms is taken in a similar form as in [12]. Here the following parameters are used:

$$
\begin{aligned}
& U_{i}^{\text {sym }}\left(A_{i}, Z_{i}, T\right)=U_{i}^{\text {sym }}\left(A_{i}, Z_{i}, T=0\right)\left(1+0.5 * 10^{-4} T^{2}\right), \\
& U_{i}^{C}\left(A_{i}, Z_{i}, \beta_{i}, T\right)=U_{i}^{C}\left(A_{i}, Z_{i}, \beta_{i}, T=0\right)\left(1-10^{-2} T^{2}\right), \\
& U_{i}^{\text {Surf }}\left(A_{i}, Z_{i}, \beta_{i}, T\right)= \\
& =U_{i}^{\text {Surf }}\left(A_{i}, Z_{i}, \beta_{i}, T=0\right)\left(1+8.5 * 10^{-3} T^{2}\right) .
\end{aligned}
$$

Here, $T$ is the temperature of each fragment (see below). The decrease of the stiffness parameter $k_{i}$ with excitation energy is taken as: $k_{i}\left(E_{i}^{*}\right)=k_{i} * \exp \left[-E_{i}^{*} / E_{k}\right]$, with $E_{k}=$ 3.7 MeV.

The shell correction terms are calculated with the Strutinsky method and the two center shell model [13]. The damping of the shell corrections with excitation energy $E_{i}^{*}$ is introduced as follows:

$$
\begin{aligned}
& \delta U_{i}^{\text {shell }}\left(A_{i}, Z_{i}, \beta_{i}, E_{i}^{*}\right)= \\
& =\delta U_{i}^{\text {shell }}\left(A_{i}, Z_{i}, \beta_{i}, E_{i}^{*}=0\right) \exp \left[-E_{i}^{*} / E_{D}\right],
\end{aligned}
$$

where $E_{D}=18.5 \mathrm{MeV}$ is the damping constant. The interaction potential consists of the Coulomb interaction potential $V^{C}$ of the two uniformly charged spheroids and nuclear interaction potential [14]

$$
V_{N}=\int \rho_{L}\left(r_{1}\right) \rho_{H}\left(R-r_{2}\right) F\left(r_{1}-r_{2}\right) d r_{1} d r_{2},
$$

in the form of the double folding of Woods-Saxon nuclear densities $\rho_{i}$ of the fragments and Skyrme-type densitydependent nucleon-nucleon interaction

$$
F\left(r_{1}-r_{2}\right)=C_{0}\left[F_{i n} \frac{\rho_{0}\left(r_{1}\right)}{\rho_{00}}+F_{e x}\left(1-\frac{\rho_{0}\left(r_{1}\right)}{\rho_{00}}\right)\right] \delta\left(r_{1}-r_{2}\right),
$$

where $\rho_{0}(r)=\rho_{L}(r)+\rho_{H}(R-r), F_{\text {in,ex }}=f_{\text {in,ex }}+$ $f_{i n, e x}^{\prime}\left[\left(N_{L}-Z_{L}\right)\left(N_{H}-Z_{H}\right)\right] /\left[\left(N_{L}+Z_{L}\right)\left(N_{H}+Z_{H}\right)\right], C_{0}=$ $300 \mathrm{MeV} \mathrm{fm}{ }^{3}, f_{\text {in }}=0.09, f_{\text {ex }}=-2.59, f_{\text {in }}^{\prime}=0.42$, $f_{\text {ex }}=-0.54$ and $\rho_{00}=0.17 \mathrm{fm}^{-3}$. The nuclear densities are taken in the two-parameter Woods-Saxon form with the diffuseness parameter $a=0.51-0.56 \mathrm{fm}$ depending on the charge number of the nucleus. The interaction potential has an inner pocket and external barrier at the distances between the tips of the fragments of about (0.5-1) fm and (1.5-2) fm (in the considered region of fissioning nuclei), respectively, depending on deformations of the fragments.

The internuclear distance $R$ in Eq. (1) corresponds to the position of this pocket (minimum) $R=R_{m}\left(A_{i}, Z_{i}, \beta_{i}\right)$. The quasifission barrier $B_{q f}\left(A_{i}, Z_{i}, \beta_{i}\right)$, calculated as the difference of the potential energies at the bottom of the inner pocket $\left(R=R_{m}\right)$ and the top of the external barrier $\left(R=R_{b}\left(A_{i}, Z_{i}, \beta_{i}\right)\right)$, prevents the decay of the DNS in $R$ [11]. Note that the height of quasifission barrier decreases with charge asymmetry.

Since the thermodynamic equilibrium is postulated at scission point, the excitation energy $E^{*}\left(A_{i}, Z_{i}, \beta_{i}, R_{m}\right)$ at scission is calculated as the initial excitation energy of the fissioning nucleus $E_{C N}^{*}=E_{n}+Q\left(E_{n}\right.$ is the neutron energy) plus the difference between the potential energies of 
the fissioning nucleus $U_{C N}(A, Z, \beta)$ and the dinuclear system at the scission point $U\left(A_{i}, Z_{i}, \beta_{i}, R_{m}\right)[6]$

$$
\begin{aligned}
& E^{*}\left(A_{i}, Z_{i}, \beta_{i}, R_{m}\right)= \\
& \quad=E_{C N}^{*}+\left[U_{C N}(A, Z, \beta)-U\left(A_{i}, Z_{i}, \beta_{i}, R_{m}\right)\right] .
\end{aligned}
$$

The relative formation probability of the DNS with particular masses, charges and deformations of the fragments is calculated with the statistical approach as follows [11]:

$$
\begin{aligned}
& w\left(A_{i}, Z_{i}, \beta_{i}, E^{*}\right)= \\
& \quad=N_{0} \exp \left\{\frac{-\left[U\left(A_{i}, Z_{i}, \beta_{i}, R_{m}\right)+B_{q f}\left(A_{i}, Z_{i}, \beta_{i}\right)\right]}{T}\right\},
\end{aligned}
$$

where $N_{0}$ is the normalization factor. In Eq. (7), the temperature is calculated as $T=\sqrt{E^{*} / a}$, where $a=A / 12$ $\mathrm{MeV}^{-1}$ is the level density parameter in the Fermi-gas model. In our calculations, a single value is used for the temperature calculated at the global minimum of $U$ before the shell damping. As seen, the height $B_{q f}$ of quasifission barrier also has an impact on the yields. With increasing elongation and decreasing charge (mass) asymmetry, $B_{q f}$ decreases, the system becomes more unstable, and decays.

In order to obtain the mass distribution in fission of a particular nucleus with the mass number $A_{i}$ and charge number $Z_{i}$ one should integrate (7) over $\beta_{L}$ and $\beta_{H}$

$$
Y\left(A_{i}, Z_{i}, E^{*}\right)=N_{0} \int d \beta_{L} d \beta_{H} w\left(A_{i}, Z_{i}, \beta_{i}, E^{*}\right) .
$$

All distributions are normalized to unity. Finally, for the calculations of mass and charge distributions the following expressions are obtained:

$$
\begin{aligned}
Y\left(A_{i}\right) & =N_{0} \sum_{Z_{i}} \int d \beta_{L} d \beta_{H} w\left(A_{i}, Z_{i}, \beta_{i}, E^{*}\right), \\
Y\left(Z_{i}\right) & =N_{0} \sum_{A_{i}} \int d \beta_{L} d \beta_{H} w\left(A_{i}, Z_{i}, \beta_{i}, E^{*}\right) .
\end{aligned}
$$

Because the dynamical treatment is not explicitly performed here, we "simulate" the dynamical effects by restricting the minimum value of the quasifission barrier. In the calculations, we take into consideration only those configurations for which $B_{q f}$ is larger than $\sim 1 \mathrm{MeV}$. This condition restricts the highly deformed configurations in the $\left(\beta_{L}, \beta_{H}\right)$ plane and, correspondingly, restricts the upper limits of integration over deformations $\beta_{L, H}$. This restriction allows us to describe the experimental data rather good (see below)

Due to the excitation energy, the fission fragment can evaporate several neutrons after fission. This changes the mass distribution but not the charge distribution. To calculate the neutron multiplicity distribution, the following expression is used:

$$
<n_{i}>=\frac{E_{i}^{\prime *}}{B_{n_{i}}+2 T_{i}} .
$$

The $B_{n_{i}}$ and $2 T_{i}$ are the separation energy and the kinetic energy of the evaporated neutron, respectively. The two

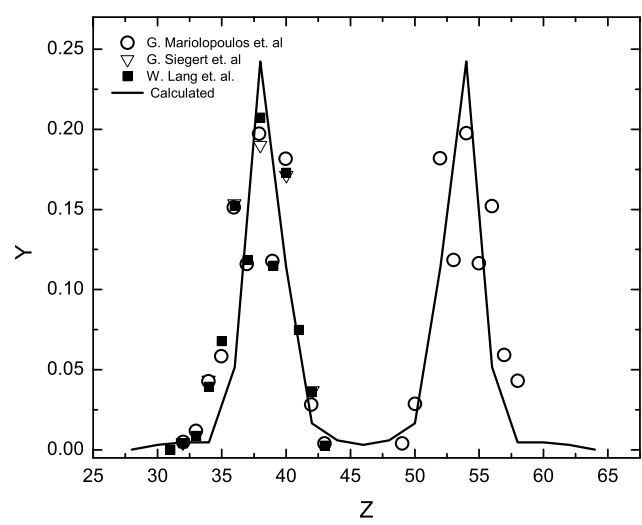

Figure 1. Calculated charge distribution compared with experimental data taken from [15-17]. The configurations allowed in the $\left(\beta_{1}, \beta_{2}\right)$ plane are chosen such that the quasifission barrier is greater than $1.15 \mathrm{MeV}$.

nuclei share the system's excitation energy proportional to their masses. Since the fragments are deformed at scission, the relaxation of the deformations to the ground state values increases the excitation energy of the nucleus by an amount equal to the deformation energy $E_{i}^{\text {def }}$

$$
E_{i}^{\prime *}=E^{*} \frac{A_{i}}{A_{L}+A_{H}}+E_{i}^{\text {def }} .
$$

In the calculations we included the post-scission neutron evaporation.

\section{Discussion of calculated results}

We perform the calculations of the charge and mass distributions of the fragments in neutron-induced fission of nuclei ${ }^{235} \mathrm{U}$ and ${ }^{238} \mathrm{U}$. As a first step, the calculations were restricted only to even-even fission fragments which mainly define the shapes of the charge and mass distributions. The inclusion of the odd-even and odd-odd fission fragments can only smooth out the distribution but can not appreciably change its shape. In order to obtain a smooth curve and to simulate the experimental uncertainty in the measurement of the mass number of the fission fragment, each calculated yield is smeared by the Gaussian with the width $\sigma_{A_{L}}=1.5 \mathrm{amu}$. The calculations were corrected for the neutron evaporation from the fragments as described in Sec. 2. We assume that the neutron emission prior to fission will not cause major change in the yield for the systems in question.

To test the model description, the calculated results are compared with the available experimental data. Our model gives a good description of the charge-yields in the fission reaction ${ }^{235} \mathrm{U}(n, \mathrm{f})$ with the thermal neutron (Fig. 1). In this reaction, the asymmetric charge (mass) distribution was observed. In Fig. 2, we show also the calculated mass distribution of fission fragments.

Our calculations reproduce the experimental data in the mass range $A_{L}=100-118$. The mass-yields for $A_{L}=94$ 100 are slightly overestimated, while the mass-yields for 


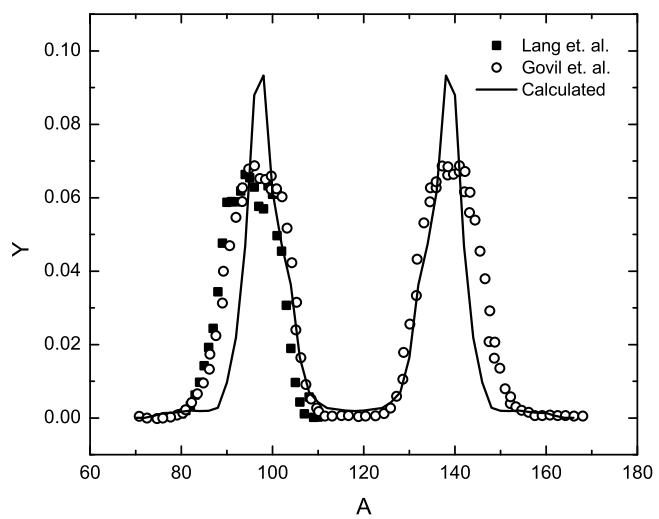

Figure 2. Calculated mass distribution compared with experimental data taken from [15], [18].
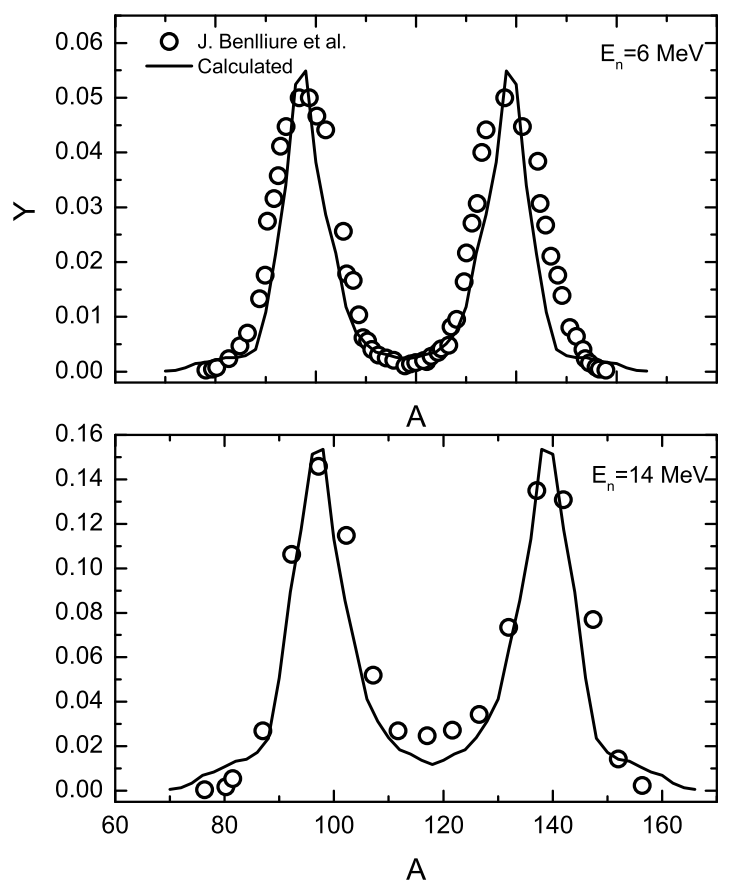

Figure 3. The calculated mass distributions (lines) for fission of ${ }^{235} \mathrm{U}$ by neutron with incident energies $6 \mathrm{MeV}$ (upper part) and $14 \mathrm{MeV}$ (lower part). The experimental data (symbols) are from Ref. [19].

$A_{L}<94$ are strongly underestimated. The latter discrepancies are unexpected because we have a reasonable good description of the charge distribution in the corresponding mass region. One can assume that the wider experimental width in Fig. 1 is due to the dynamical processes which are currently beyond the scope of the simplistic model presented here. However, in contrast to the calculated results for the thermal-neutron-induced fission the mass distributions for 6 and $14 \mathrm{MeV}$ neutron-induced fission of ${ }^{235} \mathrm{U}$ are well reproduced (Fig. 3).

The influence of the excitation energy on the peakto-valley ratio in the mass-yields is described within our model. Note that the peak-to-valley ratio in the mass yields is identical to that in the charge yields. In Fig. 4, the agreement with experimental data is shown for the isotopic
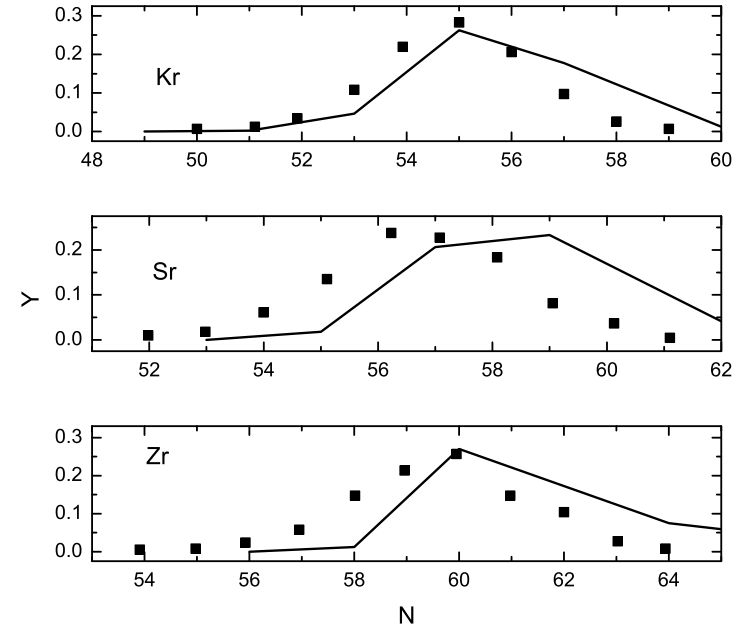

Figure 4. The calculated mass yields (lines) of the $\mathrm{Kr}, \mathrm{Sr}$, and $\mathrm{Zr}$ isotopes as a function of neutron number $N_{L}$ in fission of ${ }^{235} \mathrm{U}$ by thermal neutron. The neutron emission from the primary fragments was taken into consideration. The experimental data (symbols) are from Ref. [15].
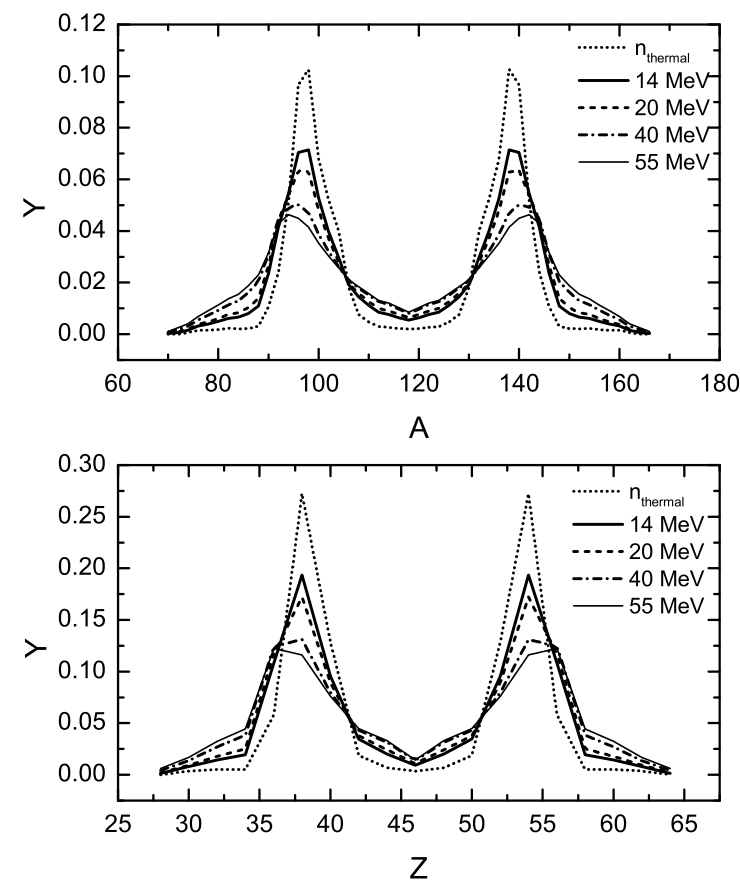

Figure 5. The calculated fragment mass (top) and charge (bottom) distributions in the ${ }^{235} \mathrm{U}(n, \mathrm{f})$ reactions at the indicated incident neutron energies.

distributions of fission fragments in the ${ }^{235} \mathrm{U}(n, \mathrm{f})$ reaction with the thermal neutrons.

To study the influence of bombarding energy (excitation energy) on the shape of the fission-fragment mass and charge distributions, we consider the reactions ${ }^{235} \mathrm{U}(n, \mathrm{f})$ at incident neutron energies $E_{n}=14,20,40$, and $55 \mathrm{MeV}$. With increasing $E_{n}$ the mass-yields (charge-yields) clearly show the decrease of asymmetric peaks and an increase of 

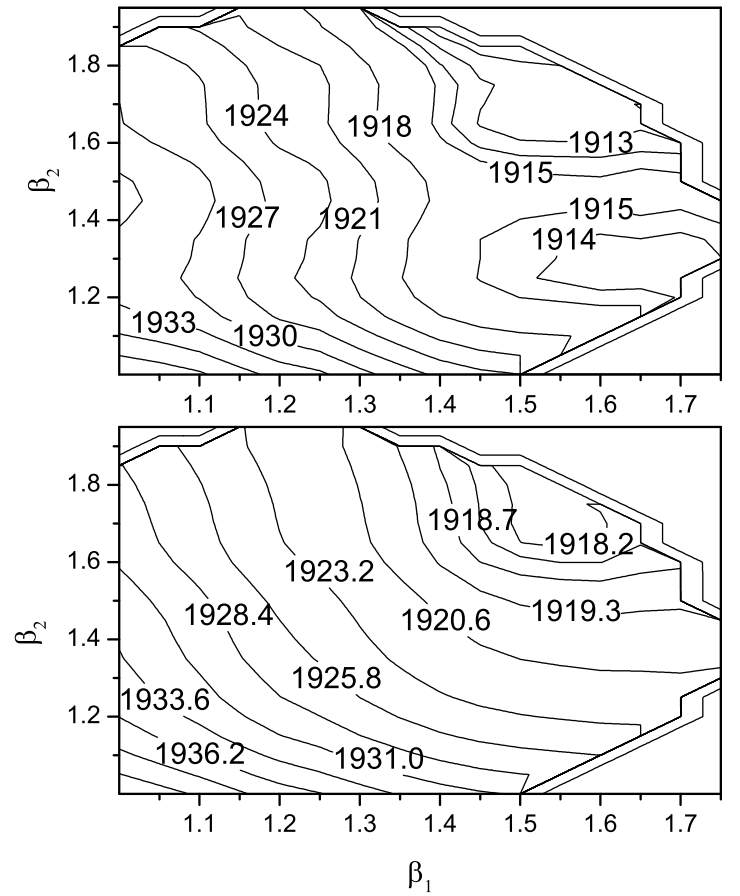

Figure 6. The potential-energy surfaces for the DNS with $\left(A_{L}, Z_{L}\right)=(96,36)$ and $\left(A_{H}, Z_{H}\right)=(140,56)$ at $E_{n}=0 \mathrm{MeV}$ (upper part) and $E_{n}=55 \mathrm{MeV}$ (lower part).

fission yields in near-symmetric mass (charge) region and very asymmetric region with $A_{L}<90\left(Z_{L}<36\right)$ (Fig. 5). The ratio between the relative yields of the fragments with $A_{L}=90-100$ and with $A_{L}=110-118$ is reduced. The increase in the $A=118$ region with neutron energy is also reported in the literature (see, e.g., [4]). The yields of near symmetric fission fragments increase nearly monotonically with incident energy from 14 to $55 \mathrm{MeV}$.

Our results indicate that the combined energydependent structures on the potential-energy surface governs the yields of the fragments. The excitation energy reduces the shell effects that causes a widening of the minima in the deformation space and a migration of the minima in the potential-energy surface (Fig. 6). As a result, the mass and charge distributions are changed (Fig. 5). However, the influence of excitation energy is not so dramatic. The mass distribution has a pronounced asymmetric shape even at the incident neutron energy $E_{n}=55 \mathrm{MeV}$ which needs the experimental verification.

In Fig. 7, the calculated fragment mass distributions are shown for the $32.8 \mathrm{MeV}$ and $45 \mathrm{MeV}$ neutron-induced fission of ${ }^{238} \mathrm{U}$. The peak-to-valley ratio and width of the two maxima are well reproduced. The explanation for the increase yield at symmetry with excitation energy is the same as in the case of ${ }^{235} \mathrm{U}(n, \mathrm{f})$.

\section{Conclusions}

The improved scission-point statistical model was employed to describe the fission reactions with actinides. The
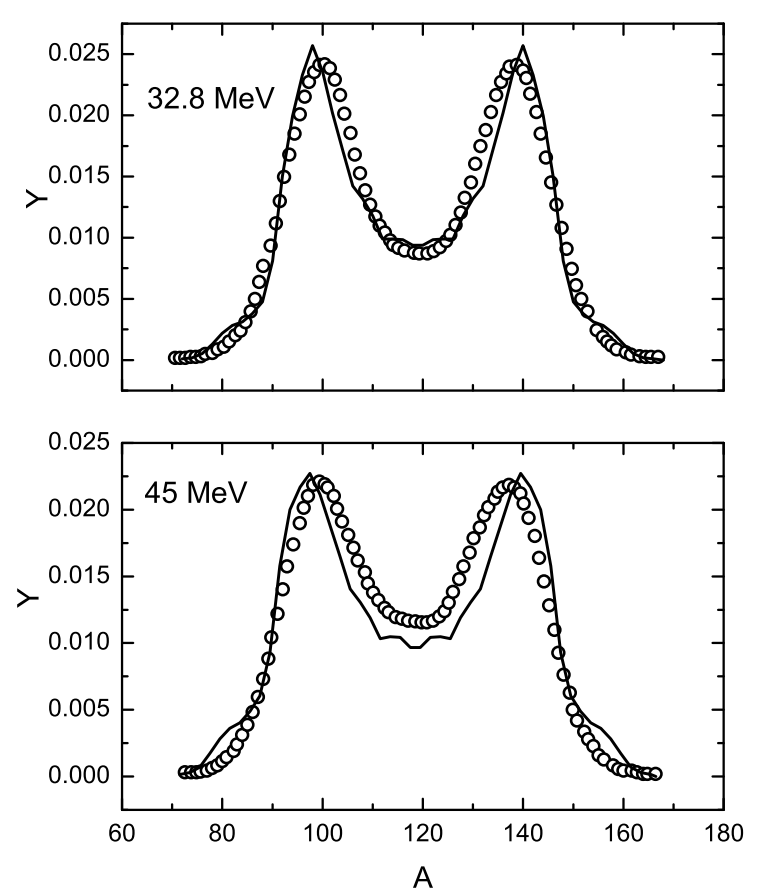

Figure 7. The calculated fragment mass distributions in the ${ }^{238} \mathrm{U}(n, \mathrm{f})$ reactions at the indicated incident neutron energies. Experimental data is taken from [20].

key element of the model is the calculation of the energy surfaces. Knowledge of the deformations of the nascent DNS at the moment of scission is crucial. The simple restriction of maximum deformations of the DNS nuclei proposed in this paper tries to mimic some dynamical effects while still retaining all the features of the statistical model. We provided a good description of the charge and mass distributions of the fission fragments in the reactions ${ }^{238} \mathrm{U}+n$ and ${ }^{235} \mathrm{U}+n$. For the latter reaction, the influence of the excitation energy on the mass and charge distribution of fission fragments for higher neutron energies, up to $55 \mathrm{MeV}$ was studied. The evolution of these fission characteristics with increasing energy was shown to be related with the widening and migration of the minima in the potential-energy surface.

The important result was the conservation of the asymmetric shapes of the mass and charge distributions of the fission fragments at high excitation energies of fissioning nucleus.

One can hope that our predictions of energydependence of the fission observables will encourage future experiments to explore the neutron-induced fission at higher bombarding energies.

\section{References}

[1] A.N. Andreyev, M. Huyse, and P. Van Duppen, Rev. Mod. Phys. 85, 1541 (2013)

[2] K.-H. Schmidt et al., Nucl. Phys. A 665, 221 (2000); K.-H. Schmidt et al., Nucl. Phys. A 693, 169 (2001) 
[3] A.N. Andreyev et al., Phys. Rev. Lett. 105, 252502 (2010)

[4] U. Brosa, S. Grossmann, and A. Müller, Phys. Rep. 197, 167 (1990)

[5] B.D. Wilkins, E.P. Steinberg, and R.R. Chasman, Phys. Rev. C 14, 1832 (1976)

[6] A.V. Andreev, G.G. Adamian, N.V. Antonenko, S.P. Ivanova, and W. Scheid, Eur. Phys. J. A 22, 51 (2004); A.V. Andreev, G.G. Adamian, N.V. Antonenko, and S.P. Ivanova, Eur. Phys. J. A 26, 327 (2005)

[7] M. Warda, A. Staszczak, and W. Nazarewicz, Phys. Rev. C 86, 024601 (2012)

[8] S. Panebianco, J.-L. Sida, H. Goutte, J.-F. Lemaître, N. Dubray, and S. Hilaire, Phys. Rev. C 86, 064601 (2012)

[9] A.V. Andreev, G.G. Adamian, and N.V. Antonenko, Phys. Rev. C 86, 044315 (2012); A.V. Andreev, G.G. Adamian, N.V. Antonenko, and A.N. Andreyev, Phys. Rev. C 88, 047604 (2013)

[10] J. Randrup and P. Möller, Phys. Rev. Lett. 106, 132503 (2011); Phys. Rev. C 84, 034613 (2011); P. Möller, J. Randrup, and A.J. Sierk, Phys. Rev. C 85, 024306 (2012)
[11] Sh.A. Kalandarov, G.G. Adamian, N.V. Antonenko, and W. Scheid, Phys. Rev. C 82, 044603 (2010); Phys. Rev. C 83, 054611 (2011); Sh.A. Kalandarov, G.G. Adamian, N.V. Antonenko, W. Scheid, and J.P. Wieleczko, Phys. Rev. C 84, 064601 (2011)

[12] G. Sauer, H. Chandra, U. Mosel, Nucl. Phys. A 264, 221 (1976)

[13] J. Maruhn and W. Greiner, Z. Phys. 251, 431 (1972)

[14] G.G. Adamian et al., Int. J. Mod. Phys. E 5, 191 (1996)

[15] W. Lang, H.G. Clerc, W. Wohlfarth, H. Schrader, and K.-H. Schmidt, Nucl. Phys. A 345, 34 (1980)

[16] G. Siegert, H. Wollnik, J. Grief, R. Decker, G. Fiedler, and B. Pfeiffer, Phys. Rev. C 14, 5 (1976)

[17] G. Mariolopoulos, Ch. Hamelin, J. Blachot, J.P. Boucqet, B. Brissot, J. Crancon, H. Nifenecker, and Ch. Ristori, Nucl. Phys. A 361, 213 (1981)

[18] Rekha Govil, S.S. Kapoor, D.M. Nadkarni, S.R.S. Murthy, and P.N. Rama Rao, Nucl. Phys. A 410, 458 (1983)

[19] J. Benlliure et al., Nucl. Phys. A 628, 458 (1998)

[20] I.V. Ryzhov, S.G. Yavshits, G.A. Tutin, N.V. Kovalev, A.V. Saulski, and N.A. Kudryashev, Phys. Rev. C 83, 054603 (2011) 